Supporting information

\title{
Simple and Efficient Green Light-Emitting Diodes Based on Thin Organolead Bromide Perovskite Films Via Tuning Precursor Ratios and Post-annealing Temperature
}

Xiaofei Zhao, Baohua Zhang*, Ruyan Zhao, Bing Yao, Xuejing Liu, Jun Liu and

Zhiyuan Xie*

State Key Laboratory of Polymer Physics and Chemistry, Changchun Institute of Applied Chemistry, Chinese Academy of Sciences, Changchun 130022, P. R. China 
Table S1. Comparison of our work with previous reported green PeLEDs

\begin{tabular}{|c|c|c|c|c|c|c|}
\hline \multicolumn{2}{|c|}{$[\mathrm{nm}]$} & Reference & $\begin{array}{c}\mathrm{L}_{\max } . \\
{\left[\mathrm{cd} \mathrm{m}^{-2}\right]}\end{array}$ & $\begin{array}{l}\mathrm{CE}_{\max } \\
{\left[\mathrm{cd} \mathrm{A}^{-1}\right]}\end{array}$ & $\begin{array}{c}\mathrm{PE}_{\max } \\
{\left[1 \mathrm{~m} \mathrm{~W}^{-1}\right]}\end{array}$ & $\begin{array}{c}\mathrm{EQE}_{\max } \\
{[\%]} \\
\end{array}$ \\
\hline \multirow{8}{*}{$\begin{array}{l}\text { Thin film } \\
(<=50)\end{array}$} & 30 & Our work & 6124 & 15.26 & 5.99 & 3.38 \\
\hline & 20 & {$[\mathrm{~S} 1]$} & 364 & - & - & 0.1 \\
\hline & 20 & [S2] & 1500 & - & - & 0.1 \\
\hline & $<50$ & [S3] & 2000 & - & - & 1.2 \\
\hline & $<50$ & [S4] & 946 & 0.43 & $<1$ & 0.12 \\
\hline & $<50$ & [S5] & 2503 & 4.5 & 3.5 & 1.1 \\
\hline & $<50$ & [S6] & 2335 & - & - & 0.19 \\
\hline & 50 & [S7] & 20000 & - & 4 & 0.8 \\
\hline \multirow{4}{*}{ Not given } & - & [S8] & $\sim 102$ & 0.27 & - & - \\
\hline & - & [S9] & 20 & 0.018 & - & 0.62 \\
\hline & - & [S10] & 4064 & 0.74 & - & 0.165 \\
\hline & - & [S11] & 10590 & - & 1.0 & 0.48 \\
\hline \multirow{7}{*}{$\begin{array}{l}\text { Thick } \\
\text { film } \\
(>50)\end{array}$} & $>50$ & [S12] & 417 & 0.577 & - & 0.125 \\
\hline & 140 & [S13] & 544.65 & 0.22 & 0.11 & 0.051 \\
\hline & 400 & [S14] & 104 & 42.9 & - & 8.53 \\
\hline & $>1000$ & [S15] & 21014 & 4.91 & - & 1.1 \\
\hline & 140 & {$[\mathrm{~S} 16]$} & 3868 & 0.54 & - & 0.13 \\
\hline & 400 & {$[\mathrm{~S} 17]$} & 3490 & 0.43 & 0.31 & 0.10 \\
\hline & 400 & [S18] & 7850 & 0.92 & 0.57 & 0.20 \\
\hline
\end{tabular}

It can be seen that the CE and EQE of our work is the best among the PeLEDs based on $\mathrm{MAPbBr}_{3}$ thin film, approaching to the best PeLEDs based on $\mathrm{MAPbBr}_{3}$ thick film [S14]. 
a)

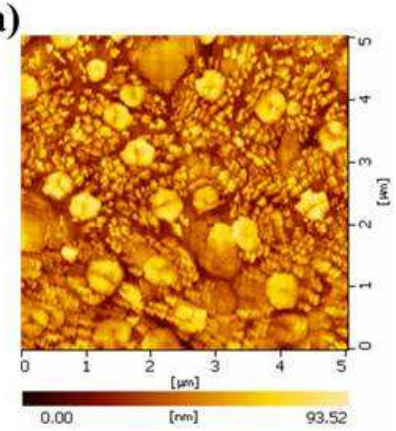

b)

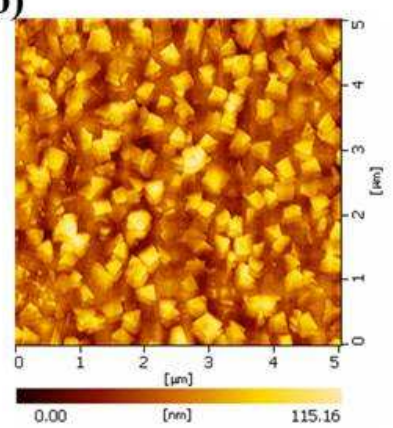

$1: 1\left(100^{\circ} \mathrm{C}\right)$

d)

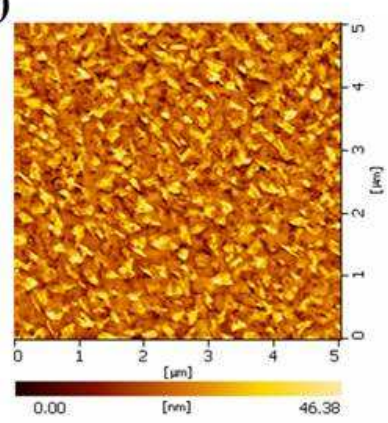

$2.2: 1\left(90^{\circ} \mathrm{C}\right)$
$1.6: 1\left(100^{\circ} \mathrm{C}\right)$

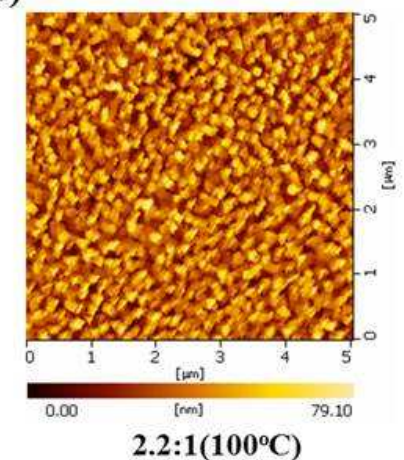

c)

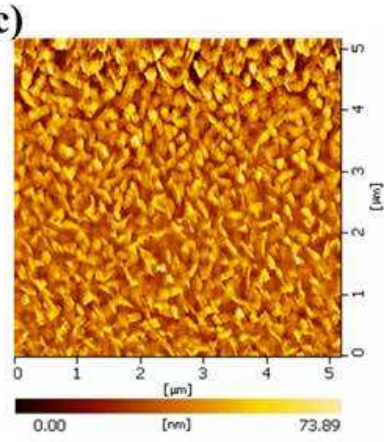

f)

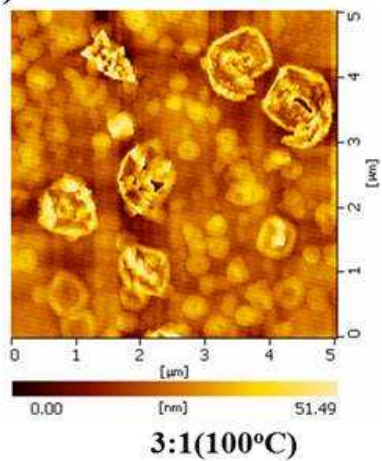

Figure S1. AFM image of the $\mathrm{MAPbBr}_{3}$ perovskite thin films with different molar ratios of $\mathrm{MABr}: \mathrm{PbBr}_{2}$ and annealing temperature. 
a)
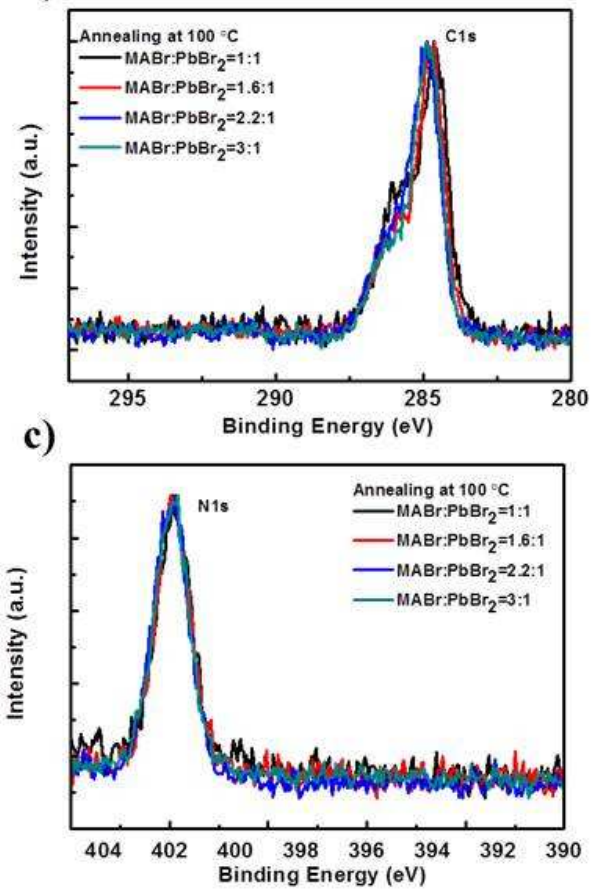

b)

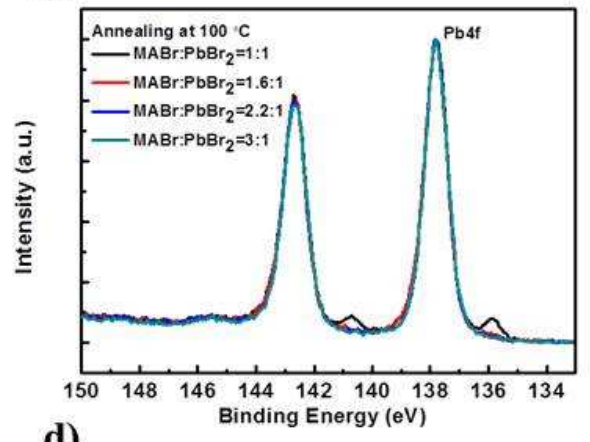

d)

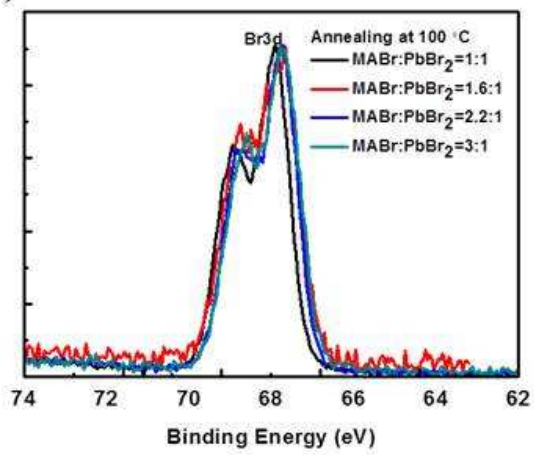

Figure S2. XPS spectra.a) C1s, b) Pb4f, c) N1s, d) Br3d spectra of $\mathrm{MAPbBr}_{3}$ layers with different molar ratios ofMABr: $\mathrm{PbBr}_{2}$. Notice that in the $\mathrm{Pb} 4 \mathrm{f}$ spectra of the perovskite film sample using $\mathrm{MABr}: \mathrm{PbBr}_{2}$ precursor ratios of $1: 1$, in addition to the two main peaks at ca. 138 and $143 \mathrm{eV}$, the two smaller peaks located at ca.136 and $141 \mathrm{eV}$ were observed, which can be assigned to metallic $\mathrm{Pb}$ signals. 


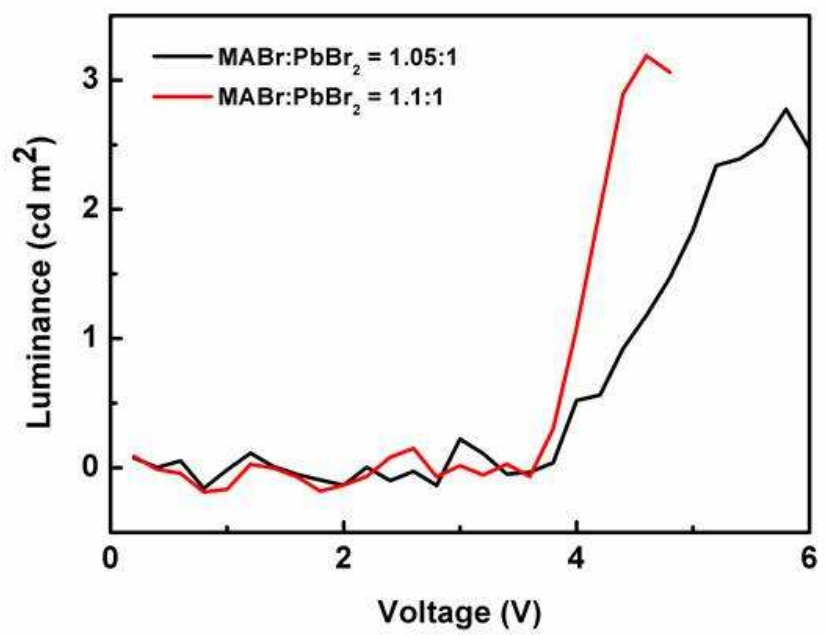

Figure S3. Plots of luminance vs. voltage of PeLEDs devices with molar ratio (1.05:1 and 1.1:1). This is different from the situation described in reported article. ${ }^{[\mathrm{S} 14]}$ 


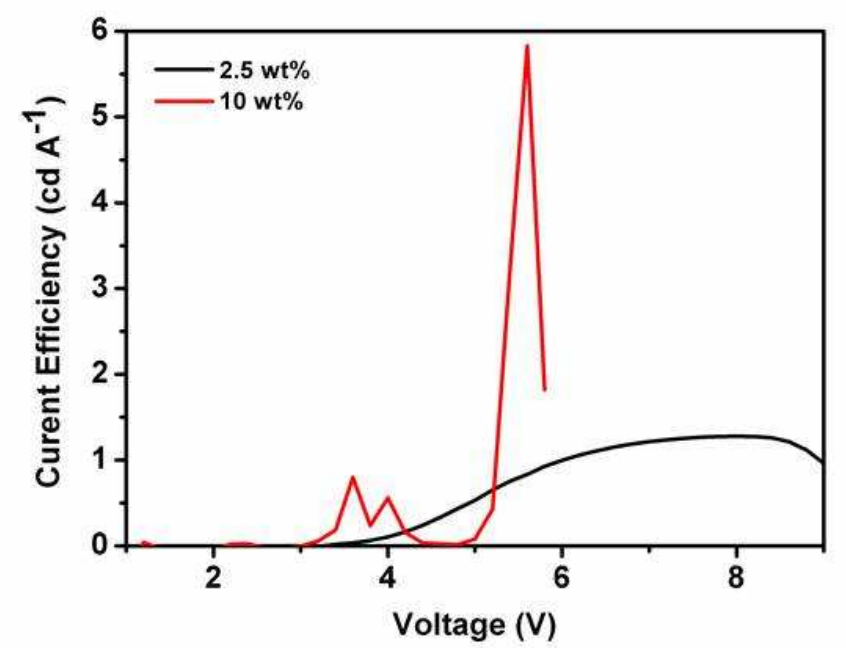

Figure S4. Plots of current efficiency vs. voltage of PeLEDs devices with different perovskites density. The different density of perovkites indicates different thickness . 
a)

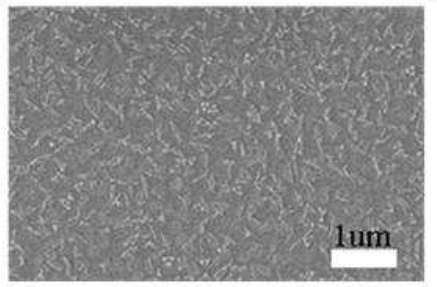

$2.2: 1\left(80^{\circ} \mathrm{C}\right)$ b)

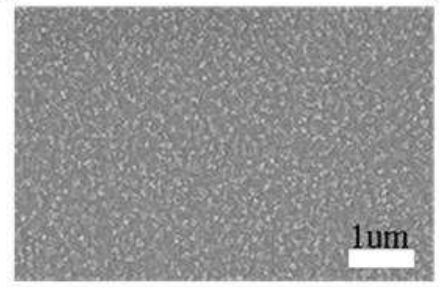

$2.2: 1\left(90^{\circ} \mathrm{C}\right)$ c)

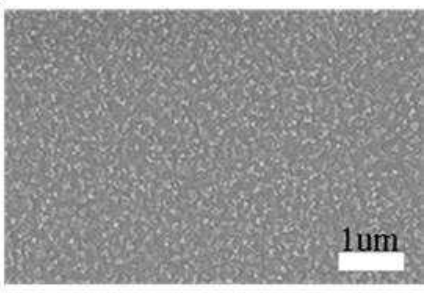

2.2:1(100 $\left.0^{\circ} \mathrm{C}\right)$

Figure S5. SEM images of $\mathrm{MAPbBr}_{3}$ layers with molar ratio of $\mathrm{MABr}: \mathrm{PbBr}_{2}=2.2: 1$ at different annealing temperature. 


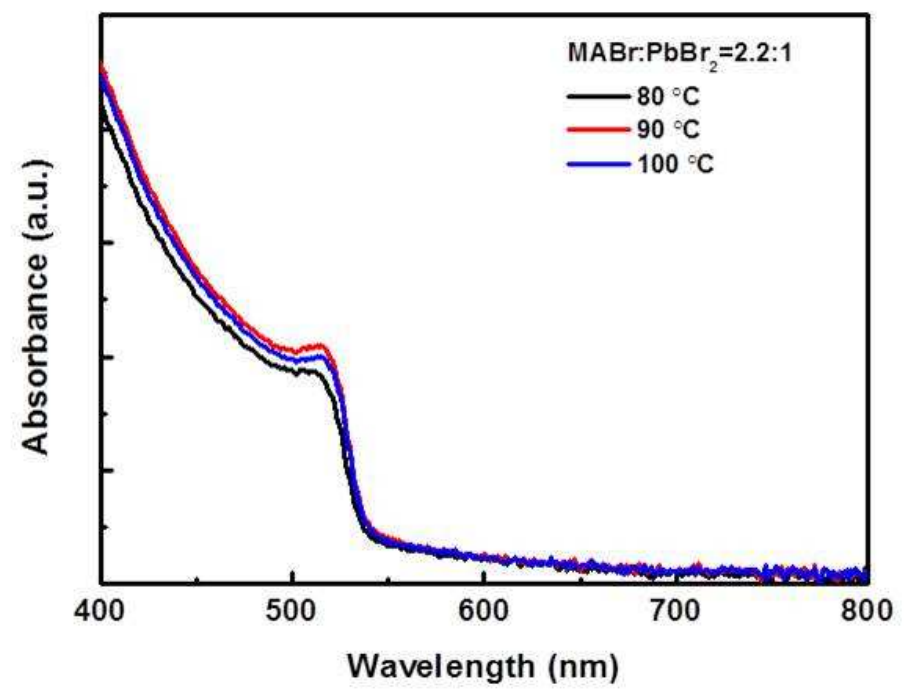

Figure S6. Absorbance of the $\mathrm{MAPbBr}_{3}$ perovskites thin films with $\mathrm{MABr}: \mathrm{PbBr}_{2}=$ 2.2:1 at different annealing temperature. 


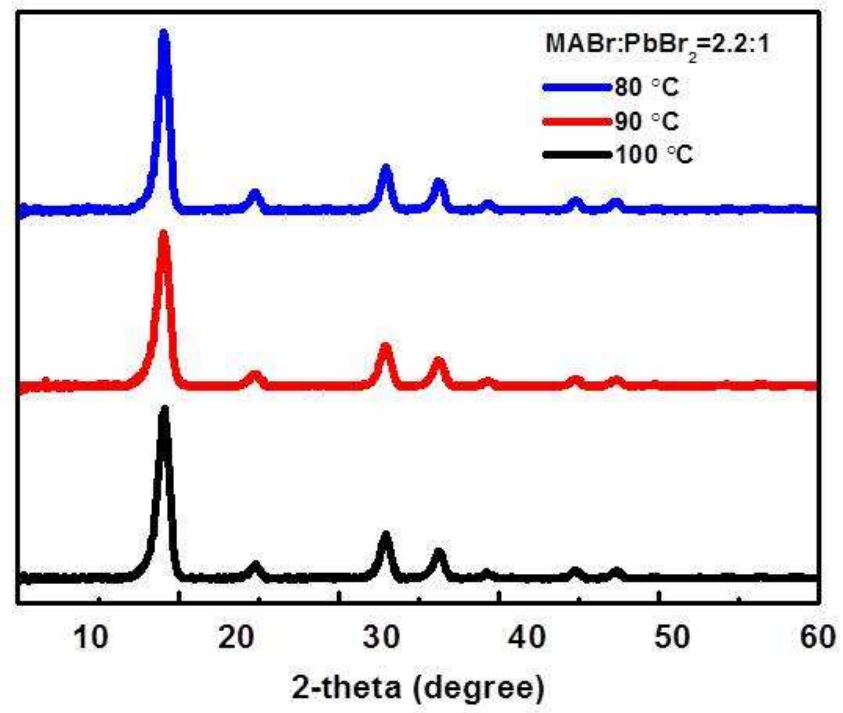

Figure S7. XRD patterns of $\mathrm{MAPbBr}_{3}$ layers with $\mathrm{MABr}: \mathrm{PbBr}_{2}=2.2: 1$ at different annealing temperature. 
a)

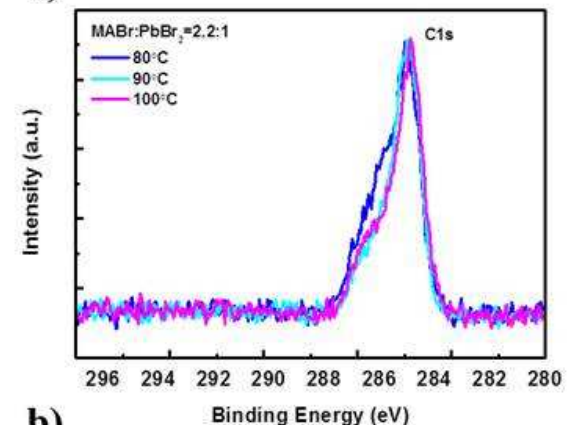

b)

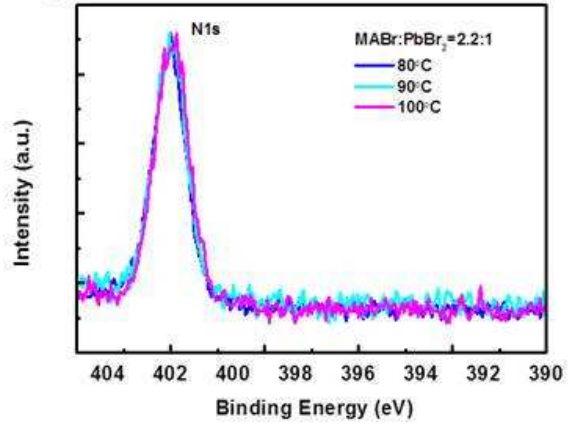

c)

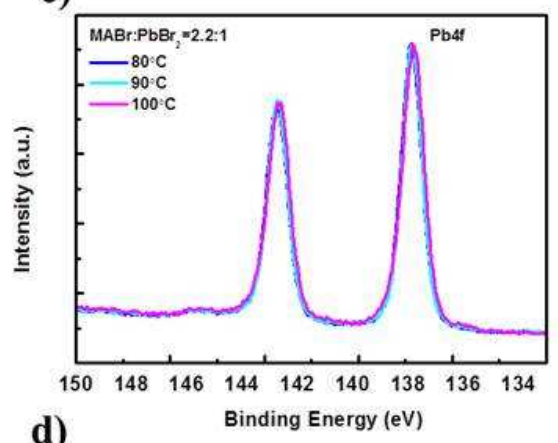

d)

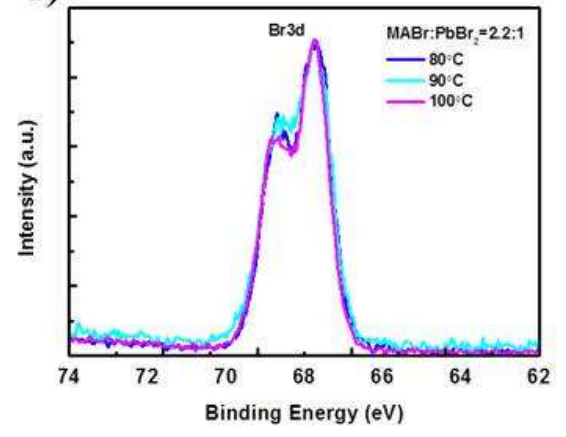

Figure S8. XPS spectra. a) C1s, b) N1s, c) Pb4f, d) $\mathrm{Br} 3 \mathrm{~d}$ spectra of $\mathrm{MAPbBr}_{3}$ layers with molar ratio $\mathrm{MABr}: \mathrm{PbBr}_{2}=2.2: 1$ at different annealing temperature. 

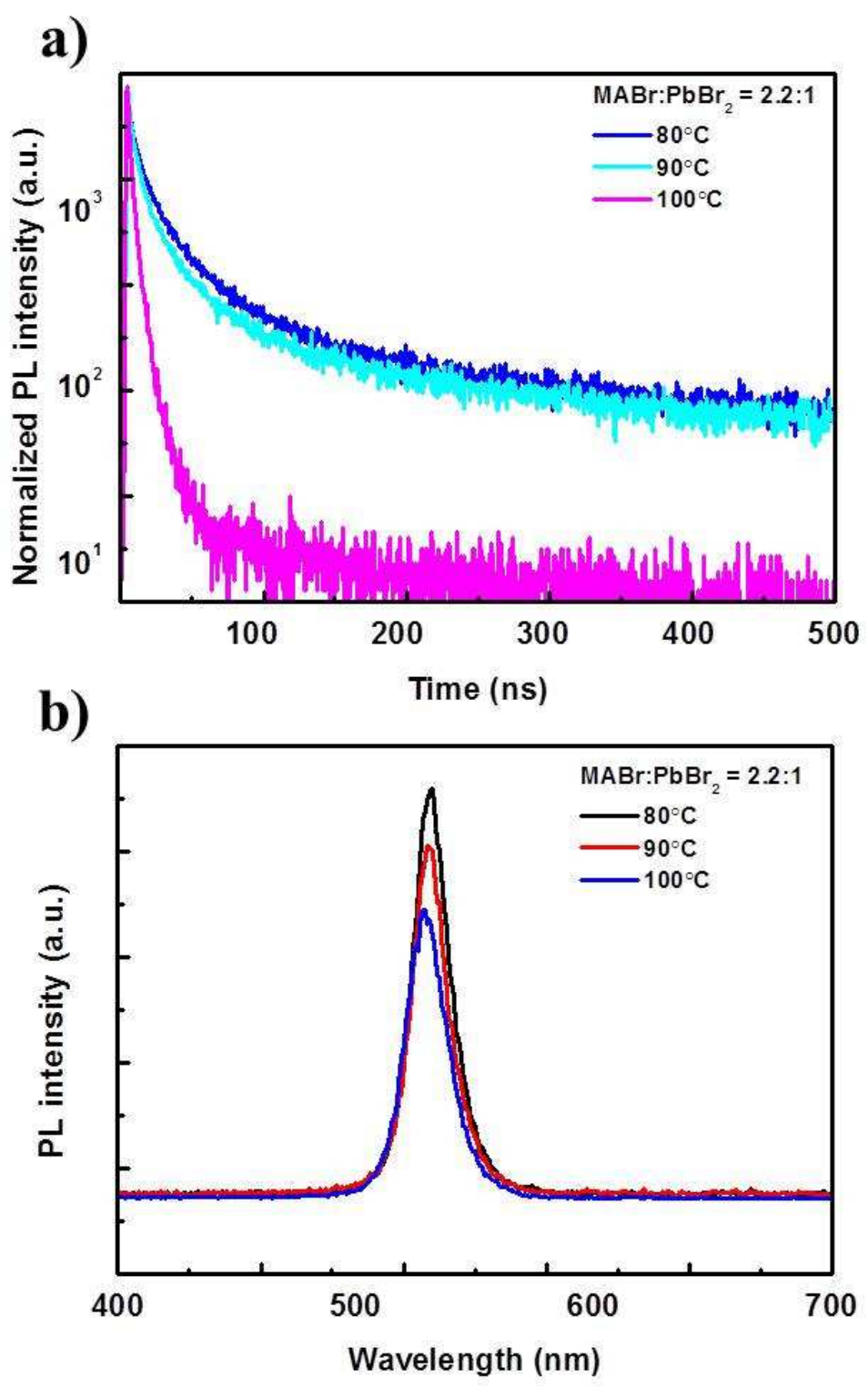

Figure S9. a) Time-resolved PL decay transient and b) Steady-state PL spectra of $\mathrm{MAPbBr} r_{3}$ layers under different annealing temperature at molar ratio of $\mathrm{MABr}: \mathrm{PbBr}_{2}$ $=2.2: 1$. 
Table S2. AverageLattice parameter and average crystallite sizes varying with molar ratio of $\mathrm{MABr}: \mathrm{PbBr}_{2}$ and different annealing temperature.

\begin{tabular}{|c|c|c|c|}
\hline $\begin{array}{c}\mathrm{MABr}: \mathrm{PbBr}_{2} \\
\text { [mol:mol] }\end{array}$ & $\begin{array}{c}\text { Temperature } \\
{\left[{ }^{\circ} \mathrm{C}\right]}\end{array}$ & $\begin{array}{l}\text { Lattice parameter } \\
\qquad[\mathrm{nm}]\end{array}$ & $\begin{array}{c}\text { Crystallite size } \\
{[\mathrm{nm}]}\end{array}$ \\
\hline $1: 1$ & 100 & & 25.6 \\
\hline $1.6: 1$ & 100 & & 26.0 \\
\hline $2.2: 1$ & 80 & 5.90 & 24.5 \\
\hline $2.2: 1$ & 90 & & 23.5 \\
\hline $2.2: 1$ & 100 & & 25.6 \\
\hline $3: 1$ & 100 & & 26.0 \\
\hline
\end{tabular}

The average lattice parameter is calculated by using Bragg's law over all peaks. The average crystallite size is calculated by using Scherrer equation over four main peaks ((100), (110), (200), (210)). 
Table S3. PL lifetimes obtained from TR-PL of $\mathrm{MAPbBr}_{3}$ under different temperature at molar ratio of $\mathrm{MABr}: \mathrm{PbBr}_{2}=2.2: 1$.

\begin{tabular}{cccccccccc}
\hline Sample & Exponential & $\tau_{1}$ & $\mathrm{~F}_{1}$ & $\tau_{2}$ & $\mathrm{~F}_{2}$ & $\tau_{3}$ & $\mathrm{~F}_{3}$ & $\tau^{2}$ & $\tau_{\text {ave. }}$ \\
(covered PMMA) & Decay fitting & {$[\mathrm{ns}]$} & {$[\%]$} & {$[\mathrm{ns}]$} & {$[\%]$} & {$[\mathrm{ns}]$} & {$[\%]$} & {$[\mathrm{ns}]$} & \\
\hline $2.2: 1\left(80^{\circ} \mathrm{C}\right)$ & Tri- & 3.46 & 10.15 & 20.28 & 43.60 & 111.32 & 46.26 & 1.147 & 60.69 \\
$2.2: 1\left(100^{\circ} \mathrm{C}\right)$ & Tri- & 2.16 & 40.78 & 7.68 & 50.18 & 129.75 & 9.04 & 1.259 & 16.45 \\
$3: 1\left(100^{\circ} \mathrm{C}\right)$ & Tri- & 1.73 & 19.42 & 11.77 & 28.83 & 118.96 & 51.74 & 1.152 & 65.3 \\
\hline
\end{tabular}

The time-resolved PL decaycurves are fitted with a tri-exponential function of time $(\mathrm{t})$ :

$$
\mathrm{A}(\mathrm{t})=\sum A_{i} e^{\frac{-t}{\tau_{i}}, \mathrm{i}}=1,2,3
$$

Where $\mathrm{A}_{\mathrm{i}}$ is prefactor and $\tau_{\mathrm{i}}$ is the time constants. ${ }^{[\mathrm{S} 11]}$ The average recombination lifetime $\left(\tau_{\mathrm{ave}}\right)$ is estimated according to the following equation:

$$
\tau_{\text {ave }}=\sum A_{i} \tau_{i}^{2} / \sum A_{i} \tau_{i}, \mathrm{i}=1,2,3
$$




\section{References}

(S1) Tan, Z.-K.; Moghaddam, R. S.; Lai, M. L.; Docampo, P.; Higler, R.; Deschler, F.; Price, M.; Sadhanala, A.; Pazos, L. M.; Credgington, D.; et al. Bright Light-Emitting Diodes Based on Organometal Halide Perovskite. Nat. Nanotechnol.2014, 9, 687-692.

(S2) Qin, X.; Dong, H.; Hu, W. Green Light-Emitting Diode from Bromine Based Organic-Inorganic Halide Perovskite. Sci. China. Mater. 2015, 58, 186-191.

(S3) Li, G.; Tan, Z.-K.; Di, D.; Lai, M. L.; Jiang, L.; Lim, J. H.-W.; Friend, R. H.; Greenham, N. C. Efficient Light-Emitting Diodes Based on Nanocrystalline Perovskite in a Dielectric Polymer Matrix. Nano Lett. 2015, 15, 2640-2644.

(S4) Song, J.; Li, J.; Li, X.; Xu, L.; Dong, Y.; Zeng, H.Quantum Dot Light-Emitting Diodes Based on Inorganic Perovskite Cesium Lead Halides (CsPbX3 ). Adv. Mater.2015, 27, 7162 .

(S5) Huang, H.; Zhao, F.; Liu, L.; Zhang, F.; Wu, X. G.; Shi, L.; Zou, B.; Pei, Q.; Zhong, H. Emulsion Synthesis of Size-Tunable $\mathrm{CH}_{3} \mathrm{NH}_{3} \mathrm{PbBr}_{3}$ Quantum Dots: An Alternative Route toward Efficient Light-Emitting Diodes. ACS Appl. Mater. Interfaces. 2015, 7, 28128.

(S6) Li, G.; Rivarola, F. W.; Davis, N. J.; Bai, S.; Jellicoe, T. C.; de la Pena, F.; Hou, S.; Ducati, C.; Gao, F.; Friend, R. H.; et al. Highly Efficient Perovskite Nanocrystal Light-Emitting Diodes Enabled by a Universal Crosslinking Method.Adv. Mater. 2016,doi: 10.1002/adma.201600064.

(S7) Wang, J.; Wang, N.; Jin, Y.; Si, J.; Tan, Z. K.; Du, H.; Cheng, L.; Dai, X.; Bai, S.; He, H.; et al. Interfacial Control Toward Efficient and Low-Voltage Perovskite Light-Emitting Diodes. Adv. Mater. 2015, 27, 2311-2316.

(S8) Hoye, R. L.; Chua, M. R.; Musselman, K. P.; Li, G.; Lai, M. L.; Tan, Z. K.; Greenham, N. C.; MacManus-Driscoll, J. L.; Friend, R. H.; Credgington, D. Enhanced Performance in Fluorene-Free Organometal Halide Perovskite Light-Emitting Diodes Using Tunable, Low Electron Affinity Oxide Electron Injectors.Adv. Mater. 2015, 27, 1414-1419. 
(S9) Kumawat, N. K.; Dey, A.; Narasimhan, K. L.; Kabra, D. Near Infrared to Visible Electroluminescent Diodes Based on Organometallic Halide Perovskites: Structural and Optical Investigation. ACS Photonics 2015, 2, 349-354.

(S10) Li, J.; Bade, S. G.; Shan, X.; Yu, Z. Single-Layer Light-Emitting Diodes Using Organometal Halide Perovskite/Poly(ethylene oxide) Composite Thin Films. Adv. Mater. 2015, 27, 5196-5202.

(S11) Ling, Y.; Yuan, Z.; Tian, Y.; Wang, X.; Wang, J. C.; Xin, Y.; Hanson, K.; Ma, B.; Gao, H. Bright Light-Emitting Diodes Based on Organometal Halide Perovskite Nanoplatelets. Adv. Mater. 2016, 28, 305-311.

(S12) Kim, Y. H.; Cho, H.; Heo, J. H.; Kim, T. S.; Myoung, N.; Lee, C. L.; Im, S. H.; Lee, T. W. Multicolored Organic/Inorganic Hybrid Perovskite Light-Emitting Diodes. Adv. Mater. 2015, 27, 1248-1254.

(S13) Yu, J. C.; Kim, D. B.; Baek, G.; Lee, B. R.; Jung, E. D.; Lee, S.; Chu, J. H.; Lee, D.-K.; Choi, K. J.; Cho, S.; et al. High-Performance Planar Perovskite Optoelectronic Devices: A Morphological and Interfacial Control by Polar Solvent Treatment. Adv. Mater. 2015, 27, 3492-3500.

(S14) Cho, H.; Jeong, S.-H.; Park, M.-H.; Kim, Y.-H.; Wolf, C.; Lee, C.-L.; Heo, J. H.; Sadhanala, A.; Myoung, N.; Yoo, S. S.; et al. Overcoming the Electroluminescence Efficiency Limitations of Perovskite Light-Emitting Diodes.Science 2015, 350, 1222-1225.

(S15) Bade, S. G.; Li, J.; Shan, X.; Ling, Y.; Tian, Y.; Dilbeck, T.; Besara, T.; Geske, T.; Gao, H.; Ma, B.; et al. Fully Printed Halide Perovskite Light-Emitting Diodes with Silver Nanowire Electrodes.ACS Nano 2016, 10, 1795-1801.

(S16) Jiao, B.; Zhu, X.; Wu, W.; Dong, H.; Xia, B.; Xi, J.; Lei, T.; Hou X.; Wu, Z.A Facile One-Step Solution Deposition via Non-Solvent/Solvent Mixture for Efficient Organometal Halide Perovskite Light-Emitting Diodes. Nanoscale, 2016, $8,11084-11090$.

(S17) Yu, J. C.; Kim, D. B.; Jung E. D.; Lee, B. R.; Song, M. H. High-Performance Perovskite Light-emitting Diodes via Morphological Control of Perovskite Film.Nanoscale, 2016, 8, 7036-7042. 
(S18) Yu, J. C.; Kim, D. W.; Kim da, B.; Jung, E. D.; Park, J. H.; Lee, A. Y.; Lee, B. R.; Di Nuzzo, D.; Friend, R. H.; Song, M. H. Improving the Stability and Performance of Perovskite Light-Emitting Diodes by Thermal Annealing Treatment.Adv. Mater. 2016, doi: 10.1002/adma.201601105. 